\title{
Research on the Evolution of Traditional Fish Pattern and Its Application in Modern Design
}

\author{
Fan Yin ${ }^{1, *}$, Jian $\mathrm{Shi}^{1}$, Xiaoqing $\mathrm{Ma}^{1}$ \\ ${ }^{1}$ Art College, Jiangxi University of Finance and Economics, Nanchang City, Jiangxi Province, 330013, China
}

\begin{abstract}
The fish pattern has a long history and it is one of the earliest traditional decorative patterns in China. The important reason for the continuous inheritance and development of fish patterns lies in its unique shape and diversified cultural connotation. Regarding the cultural meaning as the starting point, this paper makes a comprehensive and systematic discussion on the development, modeling and cultural meaning of fish patterns, and further probes into the development and application of the fish pattern in modern design from a unique perspective.
\end{abstract}

\section{Introduction}

The fish pattern is a kind of decorative pattern produced by the image of fish. It was first found in the Paleolithic age and presented in the form of fishbone ornaments. Then, fish patterns were developed and transformed in Yangshao culture and Hemudu culture, forming a variety of types, such as single fish, double fish, conjoined fish, variant fish, mermaid, fish and birds, different fish, fish and objects, and in different situations[1], different fish patterns had different parts to play. The continuous development and evolution of fish patterns also reflect the continuous progress of human society, and have an indispensable impact on design. Today, with the loss of national culture and the utilitarianism and snackification of design, the aesthetic value and cultural connotation contained in fish patterns can not only provide new design thinking for various fields of design, make this practice more personalized and humanized, but also bring the Chinese design step to a larger and wider world stage.

\section{Development of fish pattern and evolution of its shape}

\subsection{Development and evolution of fish pattern}

Throughout the long history, fish patterns have experienced continuous development and change, forming rich and unique cultural significance. With the development of different periods, fish patterns are constantly adjusting themselves and making modifications.

In ancient times, because of the awe to nature, primitive ancestors worshipped the living way and reproductive ability of fish, a kind of natural organism.
At that time, the fish patterns were simple and mysterious. Slavery society began in Xia, Shang and Zhou dynasties, and reached its peak in the Western Zhou Dynasty, during which fish patterns were mostly employed in sacrificial vessels. When it came to the Shang Dynasty, jade carvings in animal form enjoyed relative popularity and there was a custom that corpses with jade fish or jade cicadas in the mouth would be imperishable and the soul could regenerate. As a consequence, the fish-shaped sculptures made of jade were continuously produced, and the style of fish patterns also kept the simplicity and concision of ancient times, with spiritual beauty. As for the Han Dynasty, symbolic patterns began to prevail. The so-called symbolic patterns referred to phoenix, exorcism, flying immortals holding fish, etc., which represented people's yearning for a better and rich life. Most of the fish patterns in the Han Dynasty had a simple and elegant charm[2]. What is worth mentioning is the Tang tri-colored pottery in response to the Tang Dynasty due to its distinctive era characteristics, vivid appearance and affluent colors. Especially the tricolor double-fish pot, it is a classic style of Tang ceramics, with attractive fish patterns. In the Song and Yuan Dynasties, ceramics was injected rapid development and simultaneously, the patterns of fish and alga were combined together to show the scenario in which the alga swings and the fish moves. Such fish patterns look relatively elegant and unfettered. In the following Ming and Qing Dynasties, equipped with auspicious implication, fish patterns gradually got rid of the restriction given by feudal ethical code and enjoyed increasing popularity among the masses. In addition to that, fish patterns were also widely employed in producing lamps, fans and embroideries because they could not only reflect folk customs and individual livelihood, but also imply auspicious omen[3].

*Corresponding author e-mail: 1625912489@qq.com 
Table 1. Development and evolution of fish pattern.

\begin{tabular}{|c|c|c|}
\hline Periods & $\begin{array}{l}\text { Classic works on fish patterns in different } \\
\text { periods }\end{array}$ & Characteristics \\
\hline Ancient times & $\begin{array}{l}\text { Painted Pottery with Human Face\&Fish } \\
\text { Patterns }\end{array}$ & Simple, but mysterious \\
\hline Shang Dynasty & Shang-Dynasty Jade Fish & Spiritual beauty \\
\hline Han Dynasty & $\begin{array}{l}\text { Western-Han-Dynasty Coloured-drawing } \\
\text { Goose\&Fish Copper Lamp }\end{array}$ & Unsophisticated and elegant \\
\hline Tang Dynasty & Tri-colored twin fish-shaped hu & Resplendent and colorful \\
\hline $\begin{array}{l}\text { Song Dynasty and Yuan } \\
\text { Dynasty }\end{array}$ & $\begin{array}{l}\text { Yuan-dynasty Blue and White Porcelain Plate } \\
\text { with Fish and Algae Patterns }\end{array}$ & Elegant and unfettered \\
\hline $\begin{array}{l}\text { Ming Dynasty and Qing } \\
\text { Dynasty }\end{array}$ & $\begin{array}{l}\text { Blue and White Porcelain Plate with } \\
\text { Fish-changed-to-dragon Patterns }\end{array}$ & Propitious and auspicious \\
\hline
\end{tabular}

\subsection{Evolution of fish-pattern shape}

With the constant social replacement, the appearance and style of fish patterns witness evolution continuously. To begin with, people realistically depicted fish patterns according to the natural morphological features of this creature. As a result, such realistic fish patterns are of vividness and verisimilitude. As time goes by, fish patterns were summarized and further developed away from its original circumstances based on the exploration, conclusion done by creators. Correspondingly, the novel geometric fish pattern comprised by points, lines and surfaces was substituted for its original natural style and in fact, the former seemed more energetic and dynamic beauty of fluidity compared with the natural ones.

\section{Development and evolution of the cultural connotation of fish pattern}

\subsection{Reproductive worship}

Reproductive worship is a cultural phenomenon with a long history. In addition to Chinese society, it also exists in other countries, especially in India, Bhutan, Nepal, etc. In fact, it has become a religion of people. Engels once pointed out: "according to materialism, the decisive factor in history is, in the final analysis, the production and reproduction of direct life. However, the production itself can be divided into two parts. The one lies in the means of livelihood, which refers to the production of food, clothing, housing and necessary tools for such materials; the other one lies in the production of people themselves, which refers to the reproduction of species"[4]. For primitive ancestors living in the ancient times, addressing the problem associated with sufficient food and clothing was their first task. In that tough period, they lived near the water. Naturally, fish became one of the main food that they lived by and at the same time, the free breath of fish in the water also became a symbol of people's worship of nature. In addition to ensuring the most basic survival needs, the reproductive capacity and the prosperity of clans were their greatest pursuit in that period. The primitive society was in an era centered on the matrilineal clan. The primitive ancestors did not understand so much about their gender. They could only rely on the female reproductive organs for the reproduction and prosperity of the clan. Hegel also pointed out that "the view of the general reproductive capacity in nature is expressed and worshipped by the shape of male and female genitals"[5].

\subsection{Concept of auspiciousness}

With the continuous baptism of history and the improvement of civilization, people's concept of reproductive worship also changed imperceptibly. As a matter of fact, the reproductive worship was changed into a symbolic meaning of life, which was an evolution towards the concept of auspiciousness.

In the long run, fish patterns became a kind of support of people's spiritual culture. In addition to understanding the meaning of auspiciousness of fish patterns, people began to make fish patterns combined with other decorations which also represented auspiciousness, such as birds, flowers, trees and so on. By virtue of such design skills as homophonic puns, pictographic characters and their combination, people could express their wishes for auspiciousness. In modern design, we frequently witness the combination of fish patterns and lotus patterns, and they have different meanings under different circumstances. For example, as a symbol of reproductive worship, the lotus pattern would be more auspicious as it is combined with fish patterns. In fact, as early as in the poem "Jiangnan" in the Eastern Han Dynasty, people compared lotus leaves to women and fish to men, so as to describe the beautiful love between men and women. Up to now, we can still experience such kinds of combinations in wedding scenarios and associated objects. For example, the image "successive years of prosperity" employed in paper-cut, 
window decoration and ceramics is produced by virtue of the combination of patterns of lotus and fish. Compared with the implied meaning in terms of beautiful love, the image represents prosperous life year after year and has the function of exorcism. As for the allusion that we frequently mention about "carp jumps over the dragon gate", which combines the patterns of fish and dragon to describe the people who could become famous at one stroke through unremitting efforts. Such images frequently appear in the form of sculpture and are extensively employed in public art and craft works.

\section{Application of fish pattern in modern design}

\subsection{Application in architectural design}

The fish pattern has been widely used in architecture since ancient times, playing a role of fire prevention and the disaster reduction. So far, we can still see many fish shaped buildings. The "Linda sea fish square" in Beijing and the "puffer fish shaped exhibition hall" in Jiangsu Yangzhong all adopt the shape of fish patterns. Frank Gehry, a famous deconstruction design master, applied the flexible curve of fish patterns and the cultural meaning full of vitality to the architectural design and achieved great success. Among them, the Guggenheim Museum in Bilbao is the most classic. The whole appearance is composed of several fish like hyperboloids, which are covered with numerous titanium plates on the facade. From a distance, it looks like a fish scale, shining under the sun, perfectly integrated with the surrounding water in the river, which is filled with vitality.

\subsection{Application in packaging design}

The design principle of "words shall be intentional, meanings shall be auspicious" is highly respected in the design. As one of the representatives of Chinese traditional auspiciousness, the fish pattern has various auspicious implications, which is a rare design element. In the design of packaging associated with the wedding objects, the auspicious implication of celebration, blissful love and benediction to children represented by fish patterns is the most common. For example, there are fish patterns in the packaging of wedding candies, feast and tea.

\subsection{Application in product design}

The smooth and dynamic curve of fish patterns are highly sought after by product designers. In order to reduce the air resistance and improve the speed of the vehicle, the curve of fish pattern is adopted in designing the shape of automobiles and facilitates them continuously to be improved and developed into wedge shape at present. In our daily, we often come across fish-shaped products such as the comb, pen and door handle, which are more suitable for people's habit of holding. In addition to the convenience, they are more vivid and interesting from the perspective of appearance, realizing the harmony and unity of practicality and beauty.

\section{Design method of fish pattern in modern design}

Nowadays, with the economic globalization, economy and technology have been developed rapidly. Whereas, modern design also gradually loses itself in the face of novel opportunities. In order to cater to the highly developed society, there has been a great variety of similar design styles without individual and cultural characteristics. In fact, the fish pattern represents excellent Chinese traditional culture and contains unique and affluent aesthetic value and connotation, which is worth carrying forward and inheriting between designers. From the initial worship of reproduction to the later yearning for auspiciousness, the evolution of distinctive national characteristics and cultural connotation can also provide modern designers with novel design thinking and methods.

\subsection{Extracting meaning from shape}

With the continuous change of society and people's ideas, the reproductive worship of fish patterns has gradually faded. But it did not disappear completely. Instead, it began to transform to the concept of auspicious implication, so that fish patterns can continue developing in a more harmonious way. In modern design, fish patterns can be directly employed or abstractly transformed taking advantage of the professional method of extracting meanings from shapes. This practice can also be applied to various design fields and as a result, the auspicious and desirable wishes contained in fish patterns can be better expressed. Take Cybex, a high-end brand for infants and children in Germany, as an example, it introduces the "shape", "meaning" and "spirit" of bell with fish patterns into the design of its products. For this idea, designers extract the shape of these fish patters and then show them on the silver cloth of baby carriages by means of such skills as applique and embroidery. As a consequence, the expectation and yearning for a better life contained in these fish patterns are integrated into the design of baby carriages in a highly innovative way. At the same time, the fitness, energy and the cultural connotation of auspiciousness can be expressed to a greater extent.

\subsection{Deconstruction}

In order to gain a firm foothold in the new era, it is required for the fish pattern to be redesigned. By disassembling previous fish patterns and then matching the elements together, a new piece of meaningful work would be developed. This practice is called deconstruction, through which the representative elements of fish patterns could be extracted, such as the dynamic hyperbola, graceful posture, unique scales, dorsal and caudal fins as well as their special cultural 
connotation, etc. After the deconstruction, fish patterns could be shown in a novel image and what is worth mentioning is that the reconstructed image is no longer concrete, but presented in an abstract form. On the one hand, the cultural implication of fish patterns can be maintained. On the other hand, the deconstruction not only makes the cultural meaning of fish patterns better sublimated, but also provides the fish pattern with more harmonious and broad development.

\subsection{Apply the old to the new}

In this day and age, the design of fish patterns shall be paid innovation rather than rigid plagiarism. The method of applying the old to the new means that the fish pattern still makes use of the appearance of previous works, but it shall be redesigned by virtue of novel skills, materials and colors in order to make it combined with modern design to achieve the integration of tradition and modernity. Most of the buildings in China leave people a magnificent and solemn visual perception. As for this design style, fish patterns can be combined with glass materials. The visual perception of fish is dynamic and of great vitality. The combination of flexible appearance and transparent glass is beneficial for the architectural image to avoid the feeling of heaviness caused by the enormous appearance and highlights the brightness and elegance. Not only in this way, for the choice of color, we are no longer restricted by the inherent color of fish patterns and further combine the traditional color with modern ones. Based on the novel technology, these brand-new colors would be more layered and diversified.

\section{Conclusion}

The cultural connotation of fish patterns has been developed and evolved over a long period of time. The cultural belief and aesthetic value contained in them are worthy of development in the long run. With the arrival of the new era and the promotion of science and technology, the modern material civilization experiences dramatic development and people's concepts have changed extensively. It is necessary for the fish pattern to keep pace with the trend of the times so that its unique charm could be well maintained. For this purpose, designers shall combine the traditional connotation with modern thinking and make the fish pattern follow the design style with Chinese characteristics. This practice could leave more development space for fish patterns to meet the challenges of the new era.

\section{References}

1. Tao, S.Y. (1990) Changes of Chinese fish culture, Journal of Beijing Normal University (Social Sciences), 1990(02): 78-85.

2. Yin, W. (2009) Chinese fish culture. Heritage Press, Beijing.

3. Xie, Q.M. (2007) Exploration of Chinese traditional fish pattern and application of modern graphic design. Hunan Normal University. 10-18.

4. Central Compilation and Translation Bureau. (1972) Selected works of Marx and Engels, Vol. 4. People's publishing house, Beijing.

5. Hegel, G.W.F. (1979) aesthetics Volume III Volume I. The Commercial Press, Beijing. 